\title{
Tempo de aleitamento materno entre indígenas Xakriabá aldeados em Minas Gerais, Sudeste do Brasil
}

\author{
Duration of breastfeeding among the indigenous \\ Xakriabá people living in the Minas Gerais \\ state, Southeast Brazil
}

Marília Alfenas de Oliveira SíRIO'

Silvia Nascimento de FREITAS'

Adriana Maria de FIGUEIREDO ${ }^{2}$

Graziela Dutra Rocha GOUVÊA ${ }^{3}$

João Luiz PENA ${ }^{4}$

George Luiz Lins MACHADO-COELHO²

\section{R E S U M O}

\section{Objetivo}

Estimar a duração mediana do aleitamento materno na Terra Indígena Xakriabá e identificar fatores que se relacionaram ao tempo de amamentação nessa população.

\section{Métodos}

Neste estudo transversal, coletaram-se dados por meio de questionário que abrange características sociodemográficas, saúde e amamentação de 342 binômios mãe/criança, residentes na Terra Indígena Xakriabá,

1 Universidade Federal de Ouro Preto, Escola de Nutrição, Departamento de Nutrição Clínica e Social. Campus Universitário, Morro do Cruzeiro, 35400-000, Ouro Preto, MG, Brasil. Correspondência para/Correspondence to: MAO SíRIO. E-mail: $<$ mariliasirio@yahoo.com.br>

${ }^{2}$ Universidade Federal de Ouro Preto, Escola de Medicina, Departamento de Ciências Médicas. Ouro Preto, MG, Brasil.

3 Universidade Federal de Ouro Preto, Instituto de Ciências Exatas e Biológicas, Departamento de Estatística. Ouro Preto, MG, Brasil.

${ }^{4}$ Universidade Federal de Minas Gerais, Escola de Engenharia, Departamento de Meio Ambiente e Engenharia Sanitária. Belo Horizonte, MG, Brasil.

Artigo baseado na tese de MAO SÍRIO, intitulada "Estado nutricional das crianças de 0 a 12 anos e práticas alimentares da sociedade indígena Xakriabá de Minas Gerais”. Universidade Federal de Ouro Preto; 2012.

Apoio: Fundação de Amparo à Pesquisa do Estado de Minas Gerais (CDS-APQ 01161-10) e Conselho Nacional de Desenvolvimento Científico e Tecnológico (Ação sobre saúde indígena - 402530/2008-0). 
Minas Gerais, em 2007. Dados descritivos foram obtidos de 317 crianças que mamaram por pelo menos um dia, mas, para alcance dos objetivos, analisaram-se somente questionários que foram integralmente respondidos e que se referiam a crianças que mamaram por pelo menos um dia, ou seja, 82,2\% (287) do universo das 349 crianças indígenas menores de 3 anos, pelo método de Kaplan Meier e modelo de Regressão de Cox.

\section{Resultados}

A maioria das crianças era do sexo masculino $(52,0 \%), 1^{\circ}, 2^{\circ}$ ou $3^{\circ}$ filho $(53,6 \%)$ e teve o leite materno como primeiro alimento $(94,6 \%)$. A duração mediana do aleitamento materno exclusivo e do aleitamento materno foi de 11,73 meses e 7,27 dias, respectivamente, sendo os meninos e as crianças nascidas na $4^{\text {a }}$ ordem ou adiante os mais vulneráveis ao desmame.

\section{Conclusão}

Na Terra Indígena Xakriabá, a duração mediana do aleitamento materno exclusivo foi curta, e metade das crianças deixou de ter os benefícios nutricionais, imunológicos e funcionais do leite materno em idade próxima a 12 meses, quando foram desmamadas. O sexo e a ordem de nascimento se relacionaram a essa duração, mas pesquisas poderiam ser realizadas para maior entendimento dos fatores socioculturais que se relacionaram ao tempo de amamentação nesta Terra Indígena.

Palavras-chave: Aleitamento materno. Aleitamento materno exclusivo. Índios sul-americanos.

\section{A B S T R A C T}

\section{Objective}

To estimate the median duration of breastfeeding in the Xakriabá Indigenous lands and identify the factors related to breastfeeding duration among these people.

\section{Methods}

A cross-sectional study was conducted, and data regarding health, breastfeeding, and socio-demographic characteristics of 342 mothers/children living in this Indigenous Land in Minas Gerais in 2007 were collected using a questionnaire. Descriptive data of 317 children who were breastfed for at least one day were obtained, but in order to achieve this study objective, only the questionnaires that were fully completed and referred to children who were breastfed for at least one day were analyzed, i.e., 82.2\% (287 children) of the total population of indigenous children under 3 years old (349). Data were analyzed using the Kaplan Meier method and the Cox regression model.

\section{Results}

The majority of children were male $(52.0 \%), 53.6 \%$ were the $1^{\text {st }}, 2^{\text {nd }}$, or $3^{\text {rd }}$ born child, and $94.6 \%$ were breastfed as the first form of nutrition. The median duration of exclusive breastfeeding and non-exclusive breastfeeding was 11.73 months and 7.27 days, respectively; the boys and the $4^{\text {th }}$ or further children were weaned earlier.

\section{Conclusion}

In the Xakriabá Indigenous Land, the median duration of exclusive breastfeeding is short and half of the children were weaned at around 12 months of age, and therefore they could no longer reap the nutritional, immunological and functional benefits provided by breast milk. Gender and birth order were associated with the duration of breastfeeding, but further studies are needed to better understand the sociocultural factors related to the duration of breastfeeding in this Indigenous Land.

Keywords: Breastfeeding. Exclusive breastfeeding. South American indigenous.

\section{N T R O D U Ç Ã O}

No Brasil, a alimentação das crianças indígenas foi um fato curioso aos descobridores portugueses, pois na Europa as crianças eram amamentadas por "amas de leite" 1 . De fato, desde o nascimento e na fase de colo, as índias amamentavam seus filhos a qualquer hora, sem a preocupação da alternância de seio, do qual a criança só largava quando saciada ${ }^{2}$. Somente quando era capaz de andar é que a criança recebia a comida dos adultos, mas sem abandonar o peito 
da mãe ${ }^{3}$. Geralmente, o aleitamento materno se prolongava por mais de dois anos. Entretanto, no caso de doença grave ou morte da mãe, e se a criança fosse filha de inimigo tribal ou de outro parceiro, ela era precocemente desmamada ${ }^{4}$. Nessa época, não havia relatos de desnutrição infantil pelos viajantes e jesuítas ${ }^{3}$, ao contrário dos dados epidemiológicos atuais, que mostram elevadas taxas de morbimortalidade entre as crianças indígenas ${ }^{5}$.

Como exemplo, nos anos 2000 e 2009, as taxas de mortalidade infantil indígena foram 2,5 e 1,9 vezes, respectivamente, superiores àquelas de crianças brancas brasileiras ${ }^{6,7}$, refletindo a ampla desigualdade social entre os povos indígenas e não indígenas, evidenciada por precárias condições de saneamento básico, difícil acesso à alimentação adequada e aos serviços de saúde, que imprimem à população indígena, sobretudo às crianças, uma maior vulnerabilidade às doenças e ao óbito $5,6,8,9$. Além disso, dados do "I Inquérito Nacional de Nutrição e Saúde Indígena", realizado no Brasil em 2008 e 2009, revelaram que 51,2\% de 5397 crianças indígenas menores de 5 anos estavam anêmicas, ou seja, quase o dobro da prevalência reportada para as crianças não indígenas de mesma faixa etária ${ }^{10}$. Vale, ainda, observar que a taxa de mortalidade infantil no Brasil teve uma redução de $47,6 \%$ de 2000 a 2010 , chegando a 15,6/mil nascidos vivos, como reflexo de inúmeras estratégias implementadas no âmbito das políticas públicas na saúde, mas que não tiveram o mesmo alcance para os povos indígenas ${ }^{9,11}$.

Como uma das mais eficazes estratégias de redução da morbimortalidade e promoção da saúde infantil, o governo brasileiro, em consonância com as recomendações da Organização Mundial da Saúde, vem incentivando o aleitamento materno exclusivo até o sexto mês de vida e complementado por alimentos saudáveis até pelo menos os dois anos ${ }^{12,13}$. Essas recomendações ainda não foram plenamente atingidas no País, pois a duração do aleitamento materno pode ser influenciada por fatores socioeconômicos e cultu- rais $1,3,4,14,15$. Com relação aos povos indígenas, poucos são os estudos sobre as práticas de nutrição das crianças de diferentes etnias, sobretudo do aleitamento materno. Não se tem conhecimento se essas práticas sofrem influência da atual política de atenção à saúde da mulher e da criança do Brasil e se contribuem para melhoria dos indicadores de saúde da população infantil indígena.

Nesse contexto, os objetivos deste estudo foram estimar a duração mediana do Aleitamento Materno Exclusivo (AME) e Aleitamento Materno (AM) na Terra Indígena Xakriabá, em 2007, e identificar fatores que se relacionaram ao tempo de amamentação nesta Terra Indígena (TI).

\section{MÉ T O D O S}

\section{Desenho e população do estudo}

Este é um estudo transversal, de natureza quantitativa, com coleta de dados atuais e retrospectivos, por meio de questionário aplicado nos domicílios da TI Xakriabá, localizada na zona rural do município de São João das Missões, ao norte de Minas Gerais, região Sudeste do Brasil ${ }^{16}$. Em 2007, foi realizada pela equipe do estudo uma pesquisa censitária em todos os 1225 domicílios da TI. Naqueles onde residiam crianças menores de 3 anos, as mães foram convidadas a participar do estudo, cujo critério de inclusão foi: a) ser mãe de criança(s) menor(es) de 3 anos ( $<36$ meses); b) estar disposta a responder a um questionário para cada filho menor de 3 anos, e c) consentir sua livre participação no estudo. Assim, do universo de 349 crianças menores de 3 anos em 2007, coletaram-se dados de 342 (98\%). Foram excluídas do estudo 7 (2\%) crianças cujas mães não quiseram responder ao questionário.

Os Xakriabá constituem a maior etnia indígena em população e ocupação territorial, dentre as oito já reconhecidas no estado de Minas Gerais. Pertencem ao grupo linguístico Macro-Gê, divisão Akwên, mas falam o português ${ }^{17,18}$. Em 2007, somavam aproximadamente 6500 
habitantes vivendo em 52 aldeias, numa área territorial de 53 014,92 ha, na zona rural do município de São João das Missões, ao Norte do Estado ${ }^{16,18,19}$. Ocupavam originalmente a margem esquerda do Rio São Francisco, mas, devido às sucessivas invasões de suas terras, inicialmente pelos bandeirantes e depois por fazendeiros e posseiros, viram-se obrigados a se deslocarem para áreas mais distantes, desfavoráveis à pesca, agricultura e pecuária, devido principalmente à escassez de água e clima semiárido ${ }^{18,20}$. Além disso, sofreram forte miscigenação com brancos, negros e outros povos indígenas ao longo de sua história, sendo conhecidos como "grupo de caboclos"17,18. Entretanto, estão em constante processo de valorização de suas tradições, buscando reafirmar sua indianidade, revelada principalmente pelos aspectos religiosos e de posse coletiva da terra ${ }^{16,18}$. Com relação à saúde, desde o ano 2000, os Xakriabá são assistidos por 5 polos-base de atenção à saúde indígena, coordenados pelo Distrito Sanitário Especial Indígena Minas Gerais/Espírito Santo (DSEI MG/ES), do Ministério da Saúde, que vem enfrentando grandes desafios para melhorar o acesso aos serviços de saúde e as condições de saneamento básico, que são precárias em toda a $\mathrm{Tl}^{9,20}$.

\section{Coleta de dados}

Os dados relativos às características sociodemográficas, à saúde e à história pregressa e atual de amamentação foram obtidos por meio da aplicação, em um único momento, de um questionário semiestruturado em todos os domicílios onde residiam crianças menores de 3 anos, cujas mães aceitaram participar do estudo. Os questionários foram aplicados por 15 jovens indígenas Xakriabá, com ensino médio ou fundamental completo, intensivamente treinados e supervisionados pela equipe do estudo. Naqueles domicílios onde residiam mais de uma criança menor de 3 anos, a mãe respondeu a um questionário para cada criança. Assim, foram respondidos 342 questionários.
Sobre a criança, foram coletados os seguintes dados (variáveis independentes infantis): idade, sexo, ordem de nascimento, peso ao nascer, tempo de nascimento, primeiro alimento recebido, amamentação cruzada, doença do recém-nascido. Os dados referentes às mães (variáveis independentes maternas) foram: idade materna no início da gestação, número de consultas de pré-natal, início do pré-natal, tipo de parto, paridade, doença da mãe, escolaridade, ocupação e renda familiar. Ressalta-se que a idade materna deu origem a duas variáveis dicotômicas: IM1 (idade materna $\leq 15$ anos e >15 anos) e IM2 (idade materna $<20$ anos e $\geq 20$ anos).

Sobre as práticas de nutrição infantil, deu-se ênfase ao AME, ou seja, a criança recebe (ou recebeu) exclusivamente o leite materno, sem receber qualquer outro alimento líquido ou sólido, nem mesmo a água (exceção se faz a gotas de medicamentos), e a prática do AM, ou seja, a criança recebe (ou recebeu) leite materno direto da mama ou ordenhado, além de receber outros tipos de leite ou outros alimentos líquidos e/ou semi-sólidos ${ }^{13}$.

\section{Análise dos dados}

Para caracterizar o binômio mãe/criança, utilizaram-se dados das crianças que mamaram por pelo menos um dia, ou seja, 317 crianças (92,3\% dos questionários respondidos). Para análise dos dados, visando ao alcance dos objetivos, adotaram-se dois critérios simultâneos: a criança ter sido amamentada por pelo menos um dia e a mãe ter respondido integralmente ao questionário, o que resultou em 287 questionários. Assim, a população do estudo constou de 287 crianças, ou seja, 82,2\% do universo das 349 crianças Xakriabá menores de 3 anos, em 2007. Ressalta-se que, dos 342 questionários respondidos, foram excluídos das análises questionários de $55(16,1 \%)$ crianças, sendo $25(7,3 \%)$ de crianças que nunca mamaram e 30 (8,8\%) por ausência de uma ou mais respostas às perguntas do questionário. 
Para caracterizar a população do estudo, foram realizadas análises descritivas de frequência de eventos, por meio do programa Statistical Package for the Social Sciences (SPSS), versão 13 (SPSS Inc., Chicago, United States). Para estimar a duração mediana do AME (tempo em que 50\% das crianças já haviam recebido outros líquidos e/ou alimentos sólidos além do leite materno e $50 \%$ ainda mamavam exclusivamente) e do AM (tempo em que $50 \%$ das crianças não mais recebiam o leite materno e $50 \%$ ainda mamavam), realizaram-se análises de sobrevivência por meio do estimador não paramétrico de Kaplan-Meier, estimador de máxima verossimilhança da função de sobrevivência, utilizando-se o programa livre $R$ (The $R$ Foundation for Statistical Computing, Viena, Áustria, versão 2.15.2). A variável resposta foi o tempo até o desmame. As 156 crianças que ainda eram amamentadas foram consideradas como censura. Tal estimativa foi obtida por meio dos comandos para o software R, como descrito em Colosimo \& Giolo ${ }^{21}$.

Posteriormente, visando à identificação dos fatores que se associaram à duração do aleitamento materno, realizou-se análise multivariada, por meio do modelo semiparamétrico de Regressão de Cox, em que a resposta (variável dependente) foi o tempo até a ocorrência do desmame, ajustado pelas variáveis independentes (covariáveis) maternas e infantis, citadas na seção "Coleta de Dados". Para o ajuste do modelo de Cox, foram selecionadas as variáveis que permaneceram no modelo final, no qual a significância estatística considerada foi de 5\%. Para a utilização do modelo de Regressão de Cox, é necessário que a suposição de riscos proporcionais seja atendida. Para confirmar a proporcionalidade dos riscos, analisaram-se os resíduos de Schoenfeld ${ }^{21}$. Ressalta-se que, embora este estudo seja do tipo transversal, um período de risco constante foi atribuído a todas as crianças, o risco de desmame, o que justificou estimar a Razão de Risco (RR) pelo método de Regressão de Cox.
Este estudo foi aprovado pelo Comitê de Ética em Pesquisa da Universidade Federal de Ouro Preto (Processo n²005/58), pela Comissão Nacional de Ética em Pesquisa (Conep) (Parecer $n^{\circ}$ 902/2006 do Registro Conep n 12.827) e pela Fundação Nacional do Índio (Autorização n 73/CGEP/06). Obtiveram-se também aprovação das lideranças indígenas Xakriabá e a assinatura, pelas mães envolvidas no estudo, do Termo de Consentimento Livre e Esclarecido.

\section{RES U LTA DOS}

Do universo de 349 crianças indígenas Xakriabá menores de três anos, 92,7\% (317) mamaram por pelo menos um dia. As características

Tabela 1. Caracterização sociodemográfica das crianças menores de três anos amamentadas por pelo menos um dia. Terra Indígena Xakriabá (MG), 2007 (n=317).

\begin{tabular}{|c|c|c|}
\hline Variáveis & $\mathrm{n}$ & $\%$ \\
\hline \multicolumn{3}{|l|}{ Sexo } \\
\hline Masculino & 165 & 52,0 \\
\hline Feminino & 152 & 48,0 \\
\hline \multicolumn{3}{|l|}{ Ordem de nascimento } \\
\hline $1^{\circ}, 2^{\circ}$ e $3^{\circ}$ filho & 170 & 53,6 \\
\hline $4^{\circ}$ filho em diante & 147 & 46,4 \\
\hline \multicolumn{3}{|l|}{ Peso ao nascer } \\
\hline$<2.500 \mathrm{~g}$ & 42 & 13,2 \\
\hline$\geq 2.500 \mathrm{~g}$ & 245 & 77,3 \\
\hline Sem informação & 30 & 9,5 \\
\hline \multicolumn{3}{|l|}{ Idade gestacional ao nascer } \\
\hline A termo ${ }^{a}$ & 300 & 94,6 \\
\hline Pré e pós-termob & 17 & 5,4 \\
\hline \multicolumn{3}{|l|}{ Primeiro alimento ao nascer } \\
\hline Leite materno & 300 & 94,6 \\
\hline Outro (outro leite, água, chá) & 17 & 5,4 \\
\hline \multicolumn{3}{|l|}{ Amamentação cruzada } \\
\hline Não & 289 & 91,2 \\
\hline $\operatorname{Sim}$ & 28 & 8,8 \\
\hline \multicolumn{3}{|l|}{ Doença da criança pós-parto } \\
\hline Não & 271 & 85,5 \\
\hline Sim & 39 & 12,3 \\
\hline Sem informação & 7 & 2,2 \\
\hline
\end{tabular}

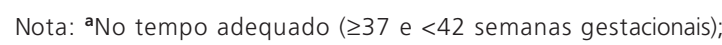
bAntes ou após o tempo adequado (40 semanas gestacionais). 
sociodemográficas dessas 317 crianças e de suas mães foram apresentadas nas Tabelas 1 e 2, respectivamente. A maioria das crianças era do sexo masculino $(52,0 \%)$, ocupava a $1^{\mathrm{a}}, 2^{\mathrm{a}}$, ou $3^{\mathrm{a}}$ ordem de nascimento e teve o leite materno como primeiro alimento (94,6\%) (Tabela 1).

Mais de $80,0 \%$ das mães eram adultas com baixa escolaridade (Tabela 2). O parto normal prevaleceu entre elas $(90,5 \%)$, e esse filho menor de três anos que participou do estudo nasceu no segundo parto ou posterior (78,2\%). Além disso, cerca de 90,0\% das mães trabalhavam sem vínculo empregatício, ou seja, realizavam trabalhos domésticos, artesanatos ou plantavam roças para subsistência da família. Nessa TI, a renda familiar mensal era igual ou inferior a um salário mínimo para a maioria das famílias (79,4\%).

O tempo mediano de amamentação foi entre 6 e 12 meses, mais próximo de 12, pois o percentual de crianças desmamadas, ou seja, que deixaram de receber o leite materno nesse tempo foi de aproximadamente $49 \%[\hat{S}(t)=0,489]$ (Tabela 3). Por interpolação linear, obteve-se o tempo mediano de 11,73 meses. Pelo mesmo método, estimou-se também a duração média do aleitamento materno, que foi de 10,088 meses (dado não mostrado).

A estimativa do tempo em que $50 \%$ das crianças continuam sendo nutridas exclusivamente pelo leite materno foi de 7,27 dias. Tem-se, ainda, um tempo médio de amamentação exclusiva estimado de 80,7 dias (dados não mostrados).

Quando foram testadas associações das covariáveis maternas e infantis com a variável dependente (tempo até a ocorrência do desmame), os fatores que se associaram à duração do aleita- mento materno foram o sexo e a ordem de nascimento das crianças (Tabela 4), sendo as

Tabela 2. Caracterização sociodemográfica das mães participantes do estudo. Terra Indígena Xakriabá (MG), 2007.

\begin{tabular}{|c|c|c|}
\hline Variáveis & $\mathrm{n}$ & $\%$ \\
\hline Idade na gestação (IM 1) & 312 & \\
\hline$\leq 15$ anos & 10 & 3,2 \\
\hline$>15$ anos & 302 & 96,8 \\
\hline Idade na gestação (IM 2) & 311 & \\
\hline$<20$ anos & 53 & 17,0 \\
\hline$\geq 20$ anos & 258 & 83,0 \\
\hline$N^{0}$ de consulta pré-natal & 307 & \\
\hline$<7$ consultas & 189 & 61,6 \\
\hline$\geq 7$ consultas & 118 & 38,4 \\
\hline Início do pré-natal (meses) & 304 & \\
\hline$\leq 3$ meses & 163 & 53,6 \\
\hline$>3$ meses & 81 & 46,4 \\
\hline Tipo de Parto & 316 & \\
\hline Normal & 286 & 90,5 \\
\hline Cirúrgico & 30 & 9,5 \\
\hline Primiparidade ${ }^{\mathrm{a}}$ & 317 & \\
\hline Sim & 69 & 21,8 \\
\hline Não & 248 & 78,2 \\
\hline Doença pós-parto & 317 & \\
\hline Não & 282 & 88,9 \\
\hline Sim & 35 & 11,1 \\
\hline Escolaridade & 305 & \\
\hline$<8$ anos & 273 & 89,5 \\
\hline$\geq 8$ anos & 32 & 10,5 \\
\hline Ocupação & 309 & \\
\hline Sem vínculo empregatício & 277 & 89,6 \\
\hline Com vínculo empregatício & 32 & 10,4 \\
\hline Renda familiar & 306 & \\
\hline$\leq 1$ salário mínimo & 243 & 79,4 \\
\hline >1 salário mínimo & 63 & 20,6 \\
\hline
\end{tabular}

Nota: aPrimiparidade: paridade do primeiro filho.

IM: Idade Materna.

Tabela 3. Tempos de sobrevivência do aleitamento materno na Terra Indígena Xakriabá (MG), 2007.

\begin{tabular}{lccccc}
\hline Tempo (meses) & $n^{\circ}$ sob risco & $n^{\circ}$ de falhas & $\hat{S}(t)$ & Erro-padrão de $\hat{S}(t)$ & IC95\% $\hat{S}(t)$ \\
\hline 3 & 277 & 25 & 0,910 & 0,0172 & $(0,877 ; 0,944)$ \\
6 & 227 & 44 & 0,733 & 0,0276 & $(0,681 ; 0,790)$ \\
12 & 126 & 42 & 0,489 & 0,0359 & $(0,423 ; 0,565)$ \\
24 & 27 & 17 & 0,181 & 0,0473 & $(0,108 ; 0,302)$ \\
35 & 3 & 3 & 0,000 & - & - \\
\hline
\end{tabular}

Nota: $\hat{S}(t)$ : Tempo de sobrevivência; IC95\%: Intervalo de Confiança de $95 \%$. 
Tabela 4. Razão de riscos de desmame, segundo as variáveis sexo e ordem de nascimento, que permaneceram no modelo final do ajuste de riscos proporcionais de Cox. Terra Indígena Xakriabá (MG), 2007.

\begin{tabular}{lccccc}
\hline Covariável & Estimativa & Erro-Padrão & Valor-p & RR & IC95\% (RR) \\
\hline Sexo & $-0,3838$ & 0,1902 & 0,043 & 0,68 & $(0,4693 ; 0,9891)$ \\
Ordem de nascimento & 0,5743 & 0,1888 & 0,002 & 1,77 & $(1,2267 ; 2,5712)$ \\
\hline
\end{tabular}

Nota: RR: Razão de Risco; IC95\%: Intervalo de Confiança de 95\%.
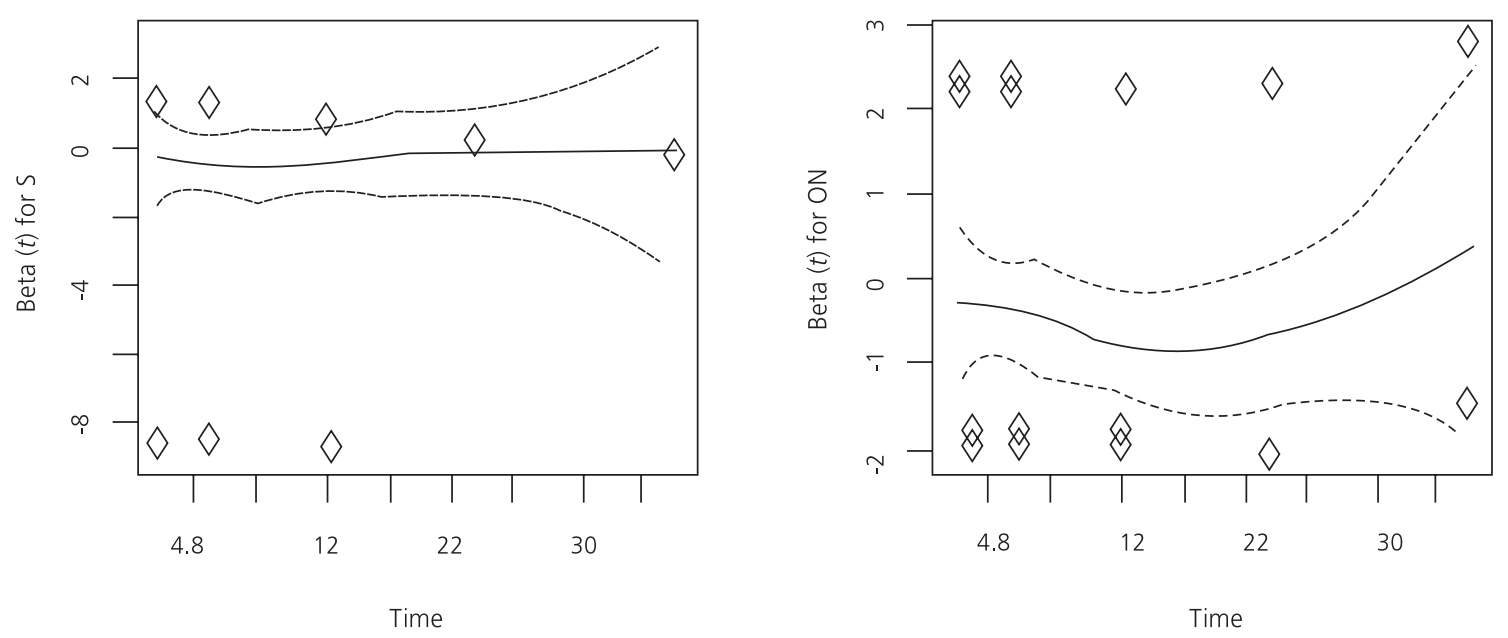

Figura 1. Resíduos padronizados de Schoenfeld versus o tempo para as covariáveis Sexo (S) e Ordem de Nascimento (ON) incluídas no modelo final.

crianças Xakriabá do sexo feminino mais protegidas do desmame do que as do sexo masculino; aquelas que ocuparam a $4^{\mathrm{a}}$ ordem de nascimento ou posterior apresentavam, aproximadamente, o dobro do risco de desmame em relação àquelas que nasceram na $1^{\text {a }}$ à $3^{\mathrm{a}}$ ordem.

Ressalta-se que, ao verificar a suposição de riscos proporcionais para cada covariável incluída no modelo final (sexo e ordem de nascimento), considerou-se que não houve evidências de violação de riscos proporcionais devido à ausência de tendências acentuadas para qualquer uma das variáveis presentes no modelo (Figura 1). A proporcionalidade também foi confirmada por meio do teste de proporcionalidade dos riscos no modelo de Cox ajustado, no qual os valores de $p$ observados para as covariáveis sexo e ordem de nascimento foram 0,724 e 0,912, respectivamente, e o valor de $p$ global foi 0,935 (dados não mostrados).

\section{I S C U S S Ã O}

Na Terra Indígena Xakriabá, iniciar a nutrição infantil pela amamentação faz parte do universo biológico e sociocultural feminino, visto que a maioria das crianças menores de três anos recebeu como primeiro alimento o leite materno. Entretanto, a duração mediana do aleitamento materno exclusivo e do aleitamento materno foi curta nesta TI. Com uma semana de vida, metade das crianças Xakriabá amamentadas passou a receber água, chás, outros tipos de leite ou outros alimentos além do leite materno, sendo, assim, 
interrompido precocemente o aleitamento materno exclusivo. Com relação à prática de aleitamento materno, metade das crianças foi desmamada em tempo muito próximo de completar um ano de vida, sendo privadas dos grandes benefícios do aleitamento materno para crescimento e desenvolvimento adequados. O desmame precoce observado neste estudo contradiz a recomendação do Ministério da Saúde, que preconiza, para todas as crianças brasileiras, o aleitamento materno exclusivo por seis meses e complementado por dois anos ou mais ${ }^{13}$.

A duração mediana de AME entre os Xakriabá foi inferior àquela observada para as crianças brasileiras não indígenas da área rural $(1,2 \text { meses })^{22}$ em 2006, e do Vale Alto Jequitinhonha $(1,5 \text { meses })^{23}$. Foi também inferior à observada para a população brasileira não indígena em 2010 (54,1 dias) ${ }^{14}$. A prática do AME também foi pouco observada em outras comunidades indígenas, como os Wari' de Rondônia, e não indígenas de área rural, que mantêm hábitos tradicionais de amamentar suas crianças, mas introduzem precocemente água, chás e outros líquidos ${ }^{24,25}$.

Uma possível explicação para essa curta duração do AME se apoiaria nos relatos de mães Xakriabá, segundo as quais a introdução precoce de água e chás é ensinada pelos mais velhos para saciar a sede, curar cólicas e complementar o leite materno. Esses mesmos motivos são também relatados pelos não indígenas ${ }^{25}$. Assim, pode-se inferir que fatores socioculturais influenciam a introdução precoce de água e chás nos primeiros meses de vida, o que reduz a duração mediana do aleitamento materno exclusivo.

Outro exemplo da influência de fatores socioculturais no aleitamento materno pode ser visto nas etnias Yaminawa, do Peru, e Yawanawa, do Brasil, no estado do Acre, que vivem em região fronteiriça. Na primeira, as práticas medicinais indígenas são muito valorizadas, e as mães, por terem pouco contato com os não indígenas nas Unidades de Saúde, amamentam exclusivamente suas crianças pequenas, continuam amamen- tando por cerca de dois anos ou mais e não usam leite em pó e mamadeiras. Já na etnia Yawanawa, do Acre, imersa num contexto sociocultural marcado por grande contato com não indígenas, muitas mulheres não amamentam seus bebês, oferecem fórmulas à base de leite em pó ou preparados à base de banana ou mandioca e lhes dão mamadeiras ${ }^{26}$. Os Xakriabá também tiveram grande contato com os não indígenas ao longo de sua história, o que poderia influenciar negativamente a prática do aleitamento materno, como visto entre os Yawanawas, do Acre. Entretanto, a focalização dessa análise na ideia de que as culturas são dinâmicas, permite flexibilizar as interações entre as culturas indígenas e não indígenas, ao contrário de vislumbrá-las como resultantes de um processo de aculturação. Nesse caso, o aleitamento materno se apresentaria como mecanismo de resistência e manutenção da cultura indígena, no contexto multiétnico da sociedade brasileira.

Considerando ainda os fatores socioculturais, no que tange às crenças passadas de geração a geração, as características do leite materno percebidas pelas mães, embora influenciadas pelas impressões de mulheres mais velhas da comunidade, podem levar à introdução de outros alimentos, como acontece entre os Wari', de Rondônia, Norte do País ${ }^{24}$. Nessa etnia, as mães acreditam na necessidade de se introduzirem alimentos não lácteos, caso o leite materno pareça "aguado". Entre os Xakriabá, essa prática também foi relatada para os bebês, para justificar a oferta frequente de chás e, mais raramente, de garapa (caldo de cana).

Com relação ao AM, a curta duração mediana observada na TI Xakriabá foi semelhante à estimada para as crianças brasileiras não indígenas da área urbana em 2006 (12,9 meses) ${ }^{22}$, inferior àquela das crianças brasileiras não indígenas da área rural (15,8 meses) ${ }^{22}$, mas superior àquela estimada para as crianças não indígenas do Vale do Jequitinhonha $\left(10,8\right.$ meses ${ }^{23}$, outra região mineira de clima semiárido e baixo Índice de Desenvolvimento Humano. Sobre o tempo de 
AM de outras etnias indígenas do Brasil, raros são os estudos quantitativos publicados até o momento. Destaca-se o estudo de Valencia et al. ${ }^{27}$, que encontrou uma duração mediana de aleitamento materno de 18,4 meses na etnia indígena Suruí, de Rondônia, região Norte do País, bem superior àquela estimada para os Xakriabá. Entre os Kamaiurá do Alto Xingu, Brasil Central, a alimentação durante o primeiro ano de vida se baseava exclusivamente no aleitamento materno e se prolongava até os dois ou três anos de ida$\mathrm{de}^{28}$. Recentemente, dados do "I Inquérito Nacional de Nutrição e Saúde Indígena" 29 revelaram elevadas taxas de amamentação, principalmente na região Norte do País, onde $90,4 \%$ das crianças ainda mamavam aos 12 meses de vida, e 51,9\% aos 24 meses. Já nas regiões Sul e Sudeste agrupadas, aos 12 meses, 75,0\% das crianças ainda mamavam, ao contrário do observado na etnia Xakriabá.

A baixa duração mediana do AME e do AM na TI Xakriabá possivelmente tem implicações nas taxas de morbimortalidade infantil, já que a criança deixa de se beneficiar, precocemente, dos componentes nutricionais, imunológicos e funcionais do leite materno, ao mesmo tempo em que se encontram vulneráveis à desnutrição e a doenças infectoparasitárias, que coexistem num ciclo sinérgico, dadas as precárias condições de saneamento básico e higiene pessoal e domiciliar a que são expostas ${ }^{9,20}$. Em 2004, a maioria das crianças Xakriabá $(75,5 \%)$ não tinha instalação sanitária nos domicílios, e 47,8\% não tinham acesso à água de poços tubulares profundos. Quanto à água de beber, 77,8\% das amostras apresentaram positividade para coliformes totais e/ou Escherichia coli9. Em 2007, época de realização deste estudo, Carvalho ${ }^{30}$ verificou que 29,9 e 2,8\% das 2081 crianças Xakriabá de 0 a 12 anos apresentaram monoparasitismo e poliparasitismo, respectivamente, por protozoários patogênicos. Além disso, 7,6\% apresentavam parasitismo por helmintos. $\mathrm{O}$ autor também relatou sobre as condições insalubres em que vive o povo Xakriabá.
Em tais condições insalubres também sobrevivem muitas crianças indígenas de outras etnias, na mesma e em outras regiões brasileiras $5^{5,8,10,24,28}$. Aliadas a isso, as mudanças nos padrões alimentares das comunidades indígenas nas últimas décadas - consequência de fatores como a restrição territorial, o esgotamento das reservas naturais, o maior acesso a alimentos industrializados, dentre outros -, têm levado à redução da diversidade alimentar e a alterações no perfil nutricional dos indígenas, aspectos em que as crianças parecem ser particularmente atingidas ${ }^{8,31}$. De fato, em 2008-2009, em duas diferentes pesquisas de base populacional, $26 \%$ das crianças indígenas menores de 5 anos apresentaram desnutrição crônica, ao contrário dos $6 \%$ reportados para crianças brasileiras (maioria não indígena) de mesma faixa etária32,33.

Quanto aos fatores que se relacionaram à duração do aleitamento materno na TI Xakriabá, as crianças do sexo feminino foram mais protegidas do desmame do que aquelas do sexo masculino, e as crianças com ordem de nascimento de $4^{\circ}$ filho adiante tiveram quase o dobro do risco de desmame, quando comparadas àquelas cujas ordens de nascimento foram a $1^{\mathrm{a}}, 2^{\mathrm{a}}$ ou $3^{a}$. Esses dados contradizem o que se esperava para populações indígenas, pois se supunha, baseando-se em Almeida', que a amamentação entre os indígenas era um híbrido natureza-cultura, em que o ato fisiológico era fortalecido pela cultura da amamentação prolongada, transmitida entre gerações e, na maioria das vezes, inviolada pela influência dos não indígenas.

$O$ fato de as meninas serem mais protegidas do desmame foi curioso, entretanto não foi possível obter mais informações para elucidar esse achado. Além disso, a amamentação prolongada poderia ser uma alternativa economicamente viável às famílias que sobreviviam com parcos recursos financeiros para aquisição de alimentos, como são as famílias Xakriabá. Nesse caso, o leite materno complementaria a alimentação oferecida à criança, protegendo-a da des- 
nutrição. Tal afirmativa é corroborada por Horta et al. ${ }^{32}$, que verificaram que a prevalência de baixo peso foi menor entre as crianças indígenas amamentadas até os 12 meses, quando comparadas àquelas de mesma idade que nunca mamaram ${ }^{32}$.

Outra possível justificativa para que a mãe pudesse amamentar por mais tempo o quarto filho ou além deste, seria a vivência da prática do AM, que interferiria positivamente na decisão materna de amamentar, bem como facilitaria o seu desempenho. Segundo estudos realizados com crianças brasileiras não indígenas, a experiência prévia de amamentação e o consequente aprendizado são determinantes importantes das altas taxas de aleitamento materno 34,35 . Entretanto, os resultados não foram ao encontro dessas perspectivas e, até o momento, não se tem explicação para a relação encontrada entre desmame, gênero e ordem e nascimento.

Neste estudo, a maioria das mães trabalha no lar, nas casas de farinha ou nas roças, ou seja, sem nenhum vínculo empregatício, o que poderia propiciar um maior contato entre mãe/filho e favorecer a amamentação. De fato, Demétrios et al. ${ }^{36}$ verificaram que o trabalho materno fora do domicílio imprime risco 1,83 vez maior para o oferecimento precoce à criança de leite de vaca e de outros alimentos diferentes do leite humano. Entretanto, entre os Xakriabá não foi observada associação entre ocupação materna e a duração do AM. Entre os Wari', as crianças tanto podiam ser levadas às roças ou casas de farinhas, local de trabalho das mães, onde seriam amamentadas, quanto podiam ficar sob o cuidado das avós ou parentes próximos, recebendo chás ou sendo amamentadas por outras mulheres (mães de (eite) ${ }^{24}$. Essa prática, denominada de amamentação cruzada, foi pouco observada entre as mães Xakriabá e não se relacionou à duração do AM.

Também não foi observada relação entre renda familiar e duração do AM, provavelmente devido ao fato de cerca de $80 \%$ das famílias sobreviverem com um salário mínimo ou até com menos, fato que as coloca em situação de semelhança econômica familiar.

Como possível limitação deste estudo, aponta-se o fato de as mães terem que relembrar a história de amamentação de seus filhos já desmamados. Entretanto, buscou-se minimizar esse possível viés de memória com treinamento intensivo dos entrevistadores indígenas nos questionamentos. Além disso, foi utilizada técnica de coleta de dados retrospectivos, que se mostrou apropriada em estudo semelhante ${ }^{15}$.

C O N CLUS Ã O

Amamentar as crianças no início da vida faz parte do universo feminino das mães Xakriabá. Entretanto, a duração mediana do AME foi curta e metade das crianças deixou de ter os benefícios nutricionais, imunológicos e funcionais do leite materno em idade próxima a 12 meses, quando foram desmamadas. Além disso, os meninos e as crianças nascidas na $4^{\text {a }}$ ordem ou adiante foram mais vulneráveis ao desmame precoce. Essa curta duração não é condizente com aquela recomendada pelo Ministério da Saúde para as crianças brasileiras. Assim, a realização de pesquisas que possibilitem o entendimento dos fatores socioculturais relacionados à curta duração do aleitamento materno nesta Terra Indígena é de grande relevância.

\section{COLABORADORES}

MAO SÍRIO participou da concepção do estudo, revisão bibliográfica, coleta, processamento, análise e interpretação dos dados, redação e revisão para aprovação final do manuscrito. SN FREITAS participou da coleta de dados e revisão crítica do manuscrito. AM FIGUEIREDO participou da concepção do estudo, da redação e revisão do manuscrito. GDR GOUVÊA participou da análise e interpretação dos dados, JL PENA e GLL MACHADO-COELHO participaram da concepção e delineamento do estudo, além da revisão crítica para aprovação final do manuscrito. 


\section{A GRADECIMENTOS}

À Bárbara dos Santos Simões, à Anabele Pires dos Santos, a Edvá Paula Vieira e a Ronaldo Veríssimo. À Fundação de Amparo à Pesquisa do Estado de Minas Gerais e Conselho Nacional de Desenvolvimento Científico e Tecnológico pelo apoio financeiro.

\section{REFERÊ N CIAS}

1. Almeida JAG. Amamentação: um híbrido naturezacultura. Rio de Janeiro: Fiocruz; 1999.

2. Ornellas LH. Alimentação através dos tempos. $3^{\text {a }}$ ed. Florianópolis: UFSC; 2003.

3. Silva AAM. Amamentação: fardo ou desejo? Estudo histórico-social dos saberes e práticas sobre aleitamento materno na sociedade brasileira [mestrado]. Ribeirão Preto: Universidade de São Paulo; 1990.

4. Rocha JM. Introdução à história da puericultura e pediatria no Brasil. In: Almeida JAG. Amamentação: um híbrido natureza cultura. Rio de Janeiro: Fiocruz; 1999. p.27-30.

5. Santos RV, Coimbra Jr. CEA. Cenário e tendências da saúde e da epidemiologia dos povos indígenas do Brasil. In: Coimbra Jr. CEA, Santos RV, Escobar $A L$, organizadores. Epidemiologia e saúde dos povos indígenas do Brasil. Rio de Janeiro: Fiocruz; 2003.

6. Brasil. Ministério da Saúde. Fundação Nacional de Saúde. Vigilância em saúde indígena: síntese dos indicadores 2010. Brasília: Funasa; 2010 [acesso 2012 abr 2]. Disponível em: http://www.paho.org/ bra/index.php?option=com_docman\&task $=$ doc download\&gid=1187\&ltemid $=423$

7. Instituto Brasileiro de Geografia e Estatística. Observações sobre a evolução da mortalidade no Brasil: o passado, o presente e perspectivas. Rio de Janeiro: IBGE; 2010 [acesso 2012 abr 2]. Disponível em: http://www.ibge.gov.br/home/estatistica/ populacao/tabuadevida/2009/notas tecnicas.pdf

8. Leite MS, Santos RV, Coimbra Jr. CEA, Gugelmin, SA. Alimentação e nutrição de povos indígenas no Brasil. In: Kac G, Sichieri R, Gigante DP, organizadores. Epidemiologia nutricional. Rio de Janeiro: Fiocruz; 2007. p.503-17.

9. Pena JL, Heller L. Saneamento e saúde indígena: uma avaliação na população Xakriabá, Minas Gerais. Eng Sanit Ambient. 2008; 13(1):222-31. http://dx.doi.org/10.1590/S1413-41522008 000100009

10. Leite MS, Cardoso AM, Coimbra Jr. CEA, Welch JR, Gugelmin SA, Lira PCl, et al. Prevalence of anemia and associated factors among indigenous children in Brazil: Results from the first national survey of indigenous people's health and nutrition. Nutrition J. 2013; 12:69. http://dx.doi.org/10.1186/ 1475-2891-12-69

11. Instituto Brasileiro de Geografia e Estatística. Censo demográfico 2010. Resultados gerais da amostra. Rio de janeiro: IBGE; 2012 [acesso 2013 ago 23]. Disponível em: http://www.ibge.gov.br/home/ presidencia/noticias/imprensa/ppts/00000 008473104122012315727483985.pdf

12. Escuder MML, Venâncio SI, Pereira JCP. Estimativa de impacto da amamentação sobre a mortalidade infantil. Rev Saúde Pública. 2003; 37(3):319-25. http://dx.doi.org/10.1590/S0034-8910200300 0300009

13. Brasil. Ministério da Saúde. Secretaria de Atenção à Saúde. Saúde da criança: nutrição infantil, aleitamento materno e alimentação complementar. Brasília: Ministério da Saúde; 2009 [acesso 2014 abr 12]. Cadernos de Atenção Básica, n 23. Disponível em: http://bvsms.saude.gov.br/bvs/ publicacoes/saude_crianca_nutricao_aleitamento_ alimentacao.pdf

14. Venâncio SI, Escuder MML, Saldiva SRDM, Giugliani ERJ. A prática do aleitamento materno nas capitais brasileiras e no Distrito Federal: situação atual e avanços. J Pediatr. 2010; 86(4):317-24. http:// dx.doi.org/10.2223/JPED.2016

15. Silveira FJF, Lamounier JA. Fatores associados à duração do aleitamento materno em três municípios na região do Alto Jequitinhonha, Minas Gerais, Brasil. Cad Saúde Pública. 2006; 22(1):69-77. http://dx.doi.org/10.1590/S0102-311X2006000 100008

16. Schettino MPF, Correia CS. Relatório circunstancial de identificação e delimitação da Terra Indígena Xakriabá Rancharia - MG. Minas Gerais: Funai; 1999. Relatório Técnico.

17. Paraíso MHB. Laudo antropológico. Identidade étnica dos Xakriabá. Belo Horizonte: arquivos do Grupo de Estudos da Educação Indígena da Faculdade de Educação da Universidade Federal de Minas Gerais; 1987.

18. Santos AFM. Do terreno dos caboclos do Sr. São João à Terra Indígena Xakriabá: as circunstâncias da formação de um povo. Um estudo sobre a construção social de fronteiras [mestrado]. Brasília: Universidade de Brasília; 1997.

19. Sistema de Informação da Atenção à Saúde Indígena. Demografia dos povos indígenas. Brasília: Funai; 2010 [acesso 2011 set 10]. Disponível em: http://sis.funasa.gov.br/transparencia_publica/ siasiweb/Layout/quantitativo_de_pessoas_2010. asp 
20. Clementino AM, Monte-Mór RL. Xakriabá: economia espaço e formação de identidade. In: XV Encontro Nacional de Estudos Populacionais; 2006 Set 18-22; Caxambu, MG. Minas Gerais: Abep; 2006 [acesso 2011 abr 18]. Disponível em: www. abep.nepo.unicamp.br/encontro2006/docspdf/ abep2006_482.pdf

21. Colosimo EA, Giolo SR. Análise de sobrevivência aplicada. São Paulo: Edgard Blücher; 2006.

22. Brasil. Ministério da Saúde. Pesquisa Nacional de Demografia e Saúde da Criança e da Mulher - PNDS 2006: dimensões do processo reprodutivo e da saúde da criança. Brasília: Ministério da Saúde; 2009 [acesso 2012 jun 3]. Disponível em: http:// bvsms.saude.gov.br/bvs/publicacoes/pnds_ crianca_mulher.pdf

23. Silveira FJF, Lamounier JA. Prevalência do aleitamento materno e práticas de alimentação complementar em crianças com até 24 meses de idade na região do Alto Jequitinhonha, Minas Gerais. Rev Nutr. 2004; 17(4):437-47. http://dx.doi.org/10.15 90/S1415-5273200400004

24. Leite, MS. Iri' Karawa, Iri' Wari': um estudo sobre as práticas alimentares e nutrição entre os índios Wari' (Pakaanova) do sudoeste da Amazônia [doutorado]. Rio de Janeiro: Fiocruz; 2004 [acesso 2012 jun 5]. Disponível em: http://bvssp.icict. fiocruz.br/pdf/leitemsd.pdf

25. Assis AMO, Prado MS, Freitas MCS, Silva RCR, Ramos B. Prática de aleitamento materno em comunidades rurais do semi-árido baiano. Rev Saúde Pública. 1994; 28(5):380-4. http://dx.doi.org/10. 1590/S0034-89101994000500012

26. Gil LP. Políticas de saúde, pluralidade terapêutica e identidade na Amazônia. Saúde Soc. 2007; 16(2):48-60. http://dx.doi.org/10.1590/S0104-12 902007000200006

27. Valencia MMA, Santos RV, Coimbra Jr. CEA, Oliveira MVG, Escobar AL. Aspectos de la fecundidad de mujeres indígenas Suruí, Rondônia, Brasil: una aproximación. Rev Bras Saúde Matern Infant. 2010; 10(3):349-58. http://dx.doi.org/10.1590/S1519-3 8292010000300008

28. Mondini L, Cano EM, Fagundes U, Lima EES, Rodrigues D, Baruzzi, RG. Condições de nutrição em crianças Kamaiurá: povo indígena do Alto Xingu, Brasil Central. Rev Bras Epidemiol. 2007; 10(1):39-47. http://dx.doi.org/10.1590/S1415-790X2007000 100005

29. Escola Nacional de Saúde Pública. Fundação Nacional de Saúde. Resultados preliminares do I
Inquérito Nacional de Nutrição e Saúde dos Povos Indígenas. Rio de Janeiro: Ensp; 2010 [acesso 2013 ago 17]. Disponível em: http://www5.ensp.fiocruz. br/biblioteca/dados/txt_919367548.pdf

30. Carvalho GLX. Prevalência de enteroparasitoses em crianças de 0 a 12 anos e condições sanitárias na Terra Indígena Xakriabá, São João das Missões, Minas Gerais, 2007 [mestrado]. Ouro Preto: Universidade Federal de Ouro Preto; 2011.

31. Coimbra CEA, Santos RV, Welch JR, Cardoso AM, Souza MC, Garnelo L, et al. The first national survey of indigenous people's health and nutrition in Brazil: Rationale, methodology, and overview of results. BMC Public Health. 2013; 13:52. http://dx.doi.org/ 10.1186/1471-2458-13-52

32. Horta BL, Santos RV, Welch JR, Cardoso AM, Santos $\mathrm{JV}$, Assis AMO, et al. Nutritional status of indigenous children: Findings from the first national survey of indigenous people's health and nutrition in Brazil. Intern J Equity Health, 2013; 12:23. http://dx.doi. org/10.1186/1475-9276-12-23

33. Brasil. Ministério do Planejamento, Orçamento e Gestão. Ministério da Saúde. Pesquisa de Orçamentos Familiares 2008-2009. Antropometria e estado nutricional de crianças, adolescentes e adultos no Brasil. Rio de Janeiro: Ministério da Saúde; 2010 [acesso 2012 jan 12]. Disponível em: http://ibge.gov.br/home/estatistica/populacao/ condicaodevida/pof/2008_2009_encaa/pof_ 20082009_encaa.pdf

34. Fujimori E, Minagawa AT, Laurenti D, Montero RMJM, Borges ANV, Oliveira IMV. Duração do aleitamento materno em menores de dois anos de idade em Itupeva, São Paulo, Brasil: há diferença entre os grupos sociais? Rev Bras Saúde Matern Infant. 2010; 10(1):39-49. http://dx.doi.org/10.15 90/S1519-38292010000100004

35. Vieira GO, Martins CC, Vieira TO, Oliveira NF, Silva, LR. Fatores preditivos da interrupção do aleitamento materno exclusivo no primeiro mês de lactação. J Pediatr. 2010; 86(5):441-4. http:// dx.doi.org/10.22 23/JPED.2010

36. Demétrio F, Pinto EJ, Assis AMO. Fatores associados à interrupção precoce do aleitamento materno: um estudo de coorte de nascimento em dois municípios do Recôncavo da Bahia, Brasil. Cad Saúde Pública. 2012; 28(4):641-54.

Recebido: junho 6, 2014 Versão final: fevereiro 20, 2015 Aprovado: março 5, 2015 